\title{
Hypertrophic Obstructive Cardiomyopathy in an Infant with Neuroblastoma
}

\author{
Sanjeev Khera ${ }^{1}$ (D) $\cdot$ H. Ravi Ramamurthy ${ }^{1} \cdot$ Randhir Ranjan $^{1} \cdot$ Aradhana Dwivedi $^{1}$
}

Received: 5 February 2021 / Accepted: 27 April 2021 / Published online: 22 May 2021

(C) Dr. K C Chaudhuri Foundation 2021

To the Editor: Hypertension (HTN) is associated with around $10 \%$ cases of neuroblastoma (NB). Cardiomyopathy associated with NB is generally dilated cardiomyopathy. Hypertrophic obstructive cardiomyopathy (HOCM) associated with NB is extremely rare and is associated with sudden death $[1,2]$.

A 5-mo-old male infant, with normal antenatal and perinatal history, presented to a tertiary care hospital with complaints of inconsolable cry and sweating over forehead. He had tachycardia, HTN, grade III ejection systolic murmer, and hepatomegaly. Echocardiography (echo) revealed HOCM, left ventricular outflow tract obstruction (LVOTO) with pressure gradient (PG) - 30/12 mm Hg and dynamic obstruction at right ventricular outflow tract (RVOT) with PG $70 \mathrm{~mm} \mathrm{Hg}$. He was started on oral propranolol. At 9 mo of age, in view of HOCM and persistent HTN despite multiple antihypertensive agents, he was referred for opinion of pediatric cardiologist.

At our center, he had paroxysmal HTN, delayed motor milestones, and severe wasting. Chest radiograph showed cardiomegaly. Electrocardiogram revealed left ventricular hypertrophy. Echo was suggestive of nonobstructive hypertrophic cardiomyopathy (HCM) as he was already on therapy with B-blockers. Ultrasonography of the abdomen done in view of persistent HTN showed right suprarenal mass measuring $3.2 \times 4.7 \times 4.2 \mathrm{~cm}$. Biopsy of the mass revealed undifferentiated neuroblastoma. The tumor did not reveal N-MYC amplification or metastasis. He was started on SIOPEN chemotherapy protocol since surgical excision was not feasible [3]. After the second course of chemotherapy, his HTN settled and more than $90 \%$ of tumor was excised. He is on monthly

Sanjeev Khera

kherakherakhera@gmail.com

1 Department of Pediatrics, Army Hospital Research \& Referral, Delhi 110010, India follow-up with growth and development monitoring, echo and is doing well.

The postulated mechanisms for cardiomyopathy in catecholamine secreting tumors are catecholamine-induced coronary and systemic vasospasm, tachycardia-associated cardiomyopathy, free radical damage, $\beta$-adrenergic receptor downregulation, and damage to sarcolemma due to calcium influx [4]. In the index child, despite a hepatomegaly (which was actually a suprarenal mass) and chronic uncontrolled HTN, USG abdomen was not done at the referring center, leading to delayed diagnosis.

Data Availability On Request.

\section{Declarations}

Conflict of Interest None.

Consent for Publication Written informed consent was obtained from the patient's father for publication of clinical details.

\section{References}

1. Kwok SY, Cheng FW, Lo AF, Leung WK, Yam MC, Li CK. Variants of cardiomyopathy and hypertension in neuroblastoma. J Pediatr Hematol Oncol. 2014;36:e158-61.

2. Maron BJ, Tajik AJ, Ruttenberg HD, et al. Hypertrophic cardiomyopathy in infants: clinical features and natural history. Circulation. 1982;65:7-17.

3. De Bernardi B, Gerrard M, Boni L, et al. Excellent outcome with reduced treatment for infants with disseminated neuroblastoma without MYCN gene amplification. J Clin Oncol. 2009;27:1034-40.

4. Sardesai SH, Mourant AJ, Sivathandon Y, Farrow R, Gibbons DO. Phaeochromocytoma and catecholamine induced cardiomyopathy presenting as heart failure. Br Heart J. 1990;63:234-7.

Publisher's Note Springer Nature remains neutral with regard to jurisdictional claims in published maps and institutional affiliations. 